\title{
Tension-induced fusion of bilayer membranes and vesicles
}

\author{
JULIAN C. SHILLCOCK AND REINHARD LIPOWSKY* \\ Max Planck Institute of Colloids and Interfaces, 14424 Potsdam, Germany \\ *e-mail: Lipowsky@mpikg.mpg.de
}

M aintaining the integrity of their protective plasma membrane is a primary requirement of cells. Accordingly, cellular events that breach the membrane are tightly regulated ${ }^{1}$. Artificial vesicles used in drug delivery must also stay intact until they have reached the desired target ${ }^{2}$. In both cases, the intrinsic resistance of the membrane to rupture must be overcome to allow the efflux of the vesicle's contents. Here, we use mesoscopic simulations ${ }^{3}$ to study the fusion of 28 -nm-diameter vesicles to $50 \times 50 \mathrm{~nm}^{2}$ planar membrane patches over $2 \mu \mathrm{s}$. We monitor the time evolution of 93 different fusion attempts. This allows us to construct a global morphology diagram, using the initial tensions of the vesicle and the planar membrane patch as control parameters, and to determine the corresponding fusion statistics. All successful fusion events are observed to occur within $350 \mathrm{~ns}$, which reflects the presence of alternative pathways for the tension relaxation.

Vesicle fusion in vivo, which involves many proteins interacting in complex ways ${ }^{4,5}$, occurs on a length scale of tens of nanometres and a timescale that is submillisecond, and possibly faster than 100 microseconds $^{6}$. It is therefore not yet possible to visualize the formation of the fusion pore experimentally. Atomistic molecular dynamics simulations are limited to length- and timescales involving perhaps 100 lipid molecules evolving for tens of nanoseconds ${ }^{7}$, leaving coarse-grained simulation as the only currently available tool to follow the molecular rearrangements that occur on the path to fusion. Accordingly, a number of simulation models have been constructed to study the process. Coarse-grained molecular dynamics simulation of the fusion ${ }^{8,9}$ of two $15-\mathrm{nm}$-diameter vesicles shows that the high curvature of the vesicles and transient protrusions of a few lipid headgroups drives their fusion. A two-dimensional model ${ }^{10}$ of fusion between rigid rod-like lipids found that an internal pressure in the ring-like vesicles was necessary for full fusion to occur. Brownian dynamics simulations ${ }^{11}$ of rigid lipids without explicit solvent revealed two possible pathways for fusion: one in agreement with the theoretical stalk model ${ }^{12,13}$, the other similar to recent Monte Carlo simulations ${ }^{14}$ of the fusion of two tense, planar membrane patches. These techniques have limitations because of the reduced dimensionality of the system ${ }^{10}$ or the lack of solvent particles ${ }^{11}$. In addition, all previous simulation studies focussed on a few fusion events, and thus could not address the statistics of fusion.

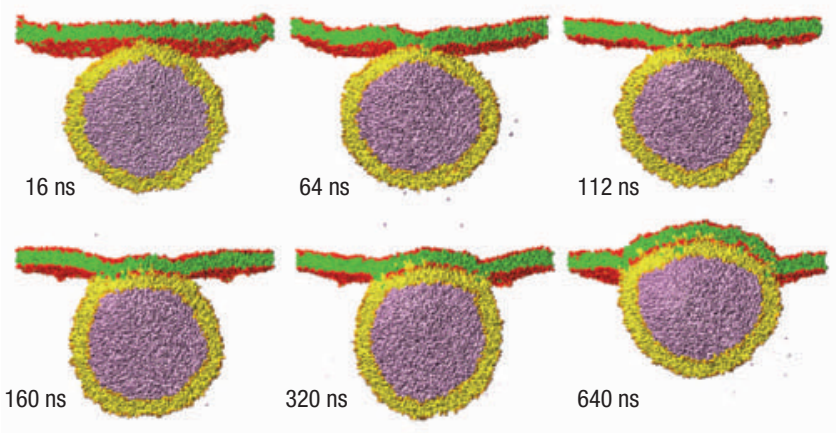

Figure 1 Adhesion of a tensionless vesicle and membrane. Time sequence of six snapshots corresponding to the adhesion of a relaxed $28 \mathrm{~nm}$ diameter vesicle and a relaxed square membrane patch, of linear extent $50 \mathrm{~nm}$, containing 6,541 and 8,227 molecules respectively. The corresponding molecular areas are $A / \mathrm{Na}_{0}{ }^{2}=1.26$ for both planar membrane and vesicle (lower left corner in Fig. 3). For each bilayer, the molecular area is obtained by dividing the area $A$ of the two bilayer water interfaces by the number $N$ of lipid molecules and by the squared bead diameter $a_{0}^{2}$. Each snapshot represents a cross-sectional image through the simulation box. Particles representing groups of water molecules initially outside the vesicle are not shown for clarity; those initially inside are shown. The slow emergence of a few water particles through the vesicle membrane is seen, which is negligible compared with the approximately 50,000 initially inside it. The thickness of the membranes is about $3.3 \mathrm{~nm}$. After initial contact between the vesicle and planar membrane during the first $64 \mathrm{~ns}$, the vesicle adheres to and spreads onto the planar membrane patch up to about $640 \mathrm{~ns}$ (note the unequal time intervals between the snapshots). After this time, the different membrane tensions are balanced and the membranes attain a fixed shape as shown in Fig. S2.A of the Supplementary Information. (All snapshots were produced using the PovRay ray tracing program, www.povray.org.)

Here, we use an alternative mesoscopic simulation technique, dissipative particle dynamics (DPD), that includes explicit solvent particles, has been shown to reproduce the correct hydrodynamic forces appropriate to a fluid ${ }^{15}$, and is capable of exploring the phase behaviour of lipid molecules ${ }^{16}$ and simulating vesicle formation ${ }^{17}$. 


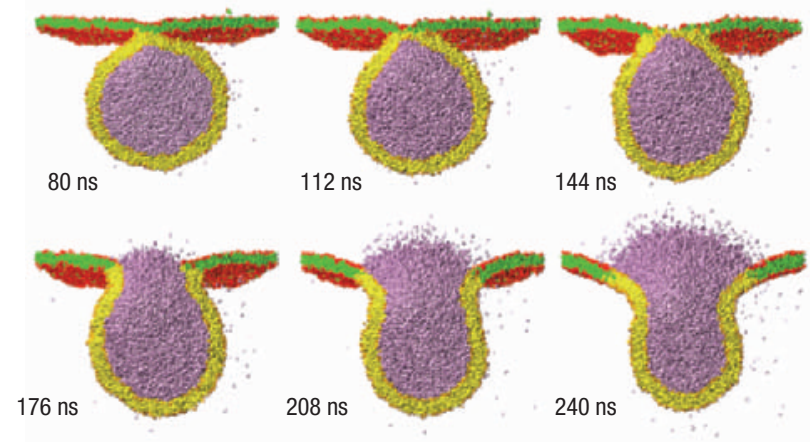

Figure 2 Fusion event between a vesicle and membrane. Time sequence of six snapshots for a typical fusion event of a tense 28 -nm-diameter vesicle and a tense square membrane patch, of linear extent $50 \mathrm{~nm}$, containing 5,684 and 5,455 lipids, respectively, driven by the uniform initial tensions in the vesicle/planar membrane. The corresponding molecular areas are $A / \mathrm{Na}_{0}{ }^{2}=1.9$ and 1.45 for the planar membrane and vesicle, respectively (upper right corner in Fig. 3). Particles representing groups of water molecules initially outside the vesicle are not shown for clarity; those initially inside the vesicle are shown. Cross-sectional images through the simulation box are shown at 32-ns intervals with the first image generated $80 \mathrm{~ns}$ after the start of the simulation. The initial contact of the vesicle to the planar membrane is by its shape fluctuations, and a few vesicle lipids are drawn across the solvent-filled gap of $1.2 \mathrm{~nm}$ into the planar membrane by its tension. The rare occurrence of a lipid spontaneously leaving the planar membrane and re-entering it is visible in the first two snapshots. The slow emergence of water particles from the vesicle interior is also visible before fusion, although the number that leave on the fusion timescale $(\sim 20)$ is again negligible compared with the number initially inside the vesicle $(\sim 50,000)$.

On monitoring the time evolution of 93 fusion attempts, we are able to construct a global morphology diagram, which summarizes the dependence of fusion on the initial tensions within the vesicle and/or planar membrane, and to determine the corresponding fusion statistics. Even in the regime of relatively large tensions, only a certain fraction of all attempts results in successful fusion events. This unreliability of tension-induced fusion indicates that one needs fine-tuned forces within the contact zone of vesicle and membrane in order to obtain a substantial increase in the fusion probability.

Methodological improvements to the original DPD algorithm ${ }^{18}$ have resulted in an improved understanding of the integration scheme and optimal integration step-size ${ }^{19}$. See ref. 20 for a general review of DPD. The main advantage over coarse-grained molecular dynamics is the ability to simulate a $50 \times 50 \times 50 \mathrm{~nm}^{3}$ fluid system for many microseconds, allowing processes such as vesicle fusion to be followed for long times. This gain in length- and timescales results from a coarse-graining of atoms (or atomic groups) into beads, which all have the same diameter $a_{0}$, and a related softening of the inter-molecular force fields to soft forces, which are all short-ranged with range $a_{0}$. A lipid is represented in our model by three linearly connected hydrophilic beads $(\mathrm{H})$, representing the polar headgroup, to which two chains of four hydrophobic beads (C) are joined. Solvent (water) particles are represented by a single bead W. Both the vesicle and planar membranes are composed of the same kind of lipid, and are in the fluid state. They can be placed under a uniform, global tension by reducing the area per molecule. These tensions are used as control parameters, and are determined using the procedure described in the Supplementary Information. All simulations take place in the $N_{\mathrm{p}} V T$ ensemble with constant particle number $N_{\mathrm{p}}$, simulation box volume $V$, and temperature $T$. All results reported

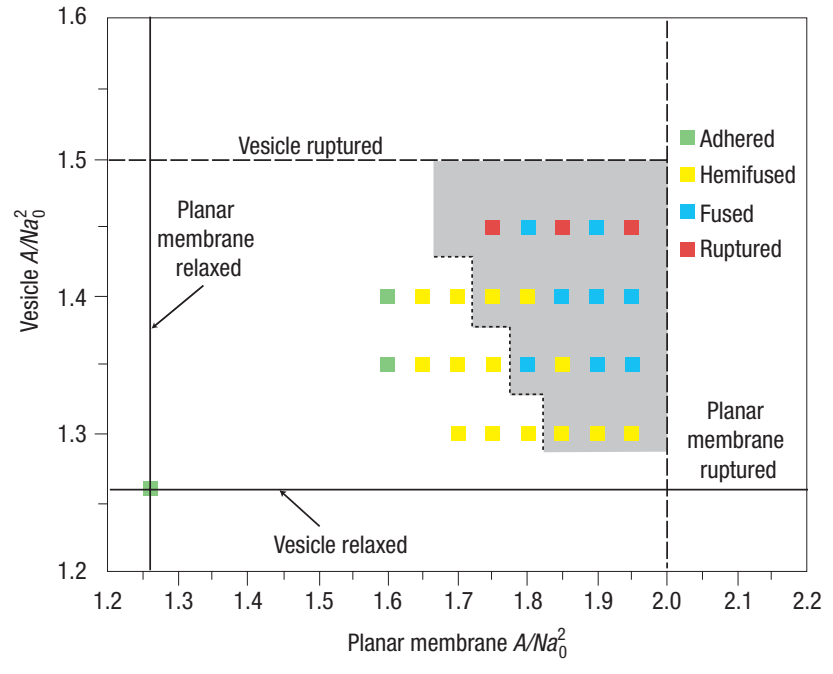

Figure 3 Morphology diagram for a vesicle in close proximity to a planar membrane. Shown as a function of the initial molecular areas, $\mathrm{ANa}^{2}$, for the planar membrane and vesicle. The tensionless states are indicated by solid lines at the lower left of the diagram, and the molecular areas, at which rupture occurs almost immediately, by dashed lines at the upper right. Because rupture is a dynamic process, occurring more slowly as the tension is reduced, the rupture boundaries represent the molecular areas at which the vesicle or planar membrane were always observed to rupture within $2 \mu$ s. The dotted line represents the stability line of hemifused states; the shaded region corresponds to the fusion regime in which we observed at least one successful fusion event. Points in the diagram are coloured according to the outcome that occurred in an absolute majority of the attempts: successful fusion is indicated by blue squares; rupture of the vesicle or planar membrane is indicated by red squares; hemifusion is indicated by yellow squares, and adhesion of the vesicle to the planar membrane, but without merging of their proximal monolayers, is indicated by green squares. Note that most squares represent the majority outcome of at least four independent simulations. Borderline cases were more intensively examined, for example, 10 runs of $1.6 \mu$ s each were performed for the point with the vesicle $A / \mathrm{Na}_{0}{ }^{2}=1.3$ and planar membrane $A / N a_{0}{ }^{2}=1.9$; and 7 runs of 640 ns for the point with vesicle $A / N a_{0}{ }^{2}=1.45$ and planar membrane $A / N a_{0}{ }^{2}=1.75$. A total of 93 independent runs were performed to generate the data represented in this diagram (see the Supplementary Information for more details about the data set.)

here were obtained at room temperature $(300 \mathrm{~K})$ in a simulation box of volume $50 \times 50 \times 50 \mathrm{~nm}^{3}$. Further details on the simulation technique are given in the Supplementary Information and ref. 3. Note that we did not attempt to map specific lipid molecules and their interactions onto the lipid-like molecules simulated here. However, because membrane fusion is a ubiquitous process observed in diverse amphiphilic systems, which have quite distinct microscopic interactions, we believe that our simulations capture the essential features that are crucial for tension-induced fusion.

Our fusion protocol starts by assembling a $50 \times 50 \mathrm{~nm}^{2}$ planar membrane patch and a 28 -nm-diameter vesicle in close proximity in a solvent-filled simulation box. The tensionless planar membrane contains approximately 8,200 lipids, whereas the tensionless vesicle contains approximately 6,500 lipids. Uniform initial tensions are induced by reducing the number of molecules for fixed projected area and fixed volume of membrane patch and vesicle, respectively.

No fusion is observed when the vesicle and planar membrane are initially relaxed. Instead, the vesicle adheres to and spreads onto the planar membrane as shown in Fig. 1. Direct inspection of this 
figure shows that the shape of both vesicle and patch changes during the spreading process until about $640 \mathrm{~ns}$. During this time period, the different membrane segments experience different tensions that are not balanced along the boundary of the contact zone, and the system exhibits hydrodynamic interactions through the water particles. After about $640 \mathrm{~ns}$, the system has relaxed and its morphology no longer changes up to 1,440 ns; see Fig. S2.A in the Supplementary Information where the time evolution of the tensions is discussed in more detail.

A successful fusion event is shown in Fig. 2 between a planar membrane containing 5,455 lipids (molecular area $A / N a_{0}{ }^{2}=1.9$ corresponding to a tension $50 \mathrm{mN} \mathrm{m}^{-1}$ ) and a vesicle containing 5,684 lipids $\left(A / N a_{0}{ }^{2}=1.45\right)$. For each bilayer, the molecular area is obtained by dividing the area $A$ of the two bilayer water interfaces by the number $N$ of lipid molecules and by the squared bead diameter $a_{0}^{2}$. The first snapshot in Fig. 2 shows the tense membrane and vesicle closely apposed, and a few lipids bridging the solvent gap. Subsequent snapshots show the development of the fusion pore through a flattened, contact zone in which the proximal monolayer lipids intermingle. We note that the timescale for the fusion event, a few hundred nanoseconds, is supported by the existence of a relatively sharp boundary separating the lipids initially in the planar membrane from those initially in the vesicle seen in the final snapshot of Fig. 2, indicating a lack of significant in-plane diffusion during the pore opening.

However, fusion is not always observed even in the presence of relatively large initial tensions because the system can follow alternative pathways to relax these tensions. The alternative pathways are provided by the rupture of the planar membrane, rupture of the vesicle membrane, and hemifusion of the two membranes in the contact zone. The hemifused state arises from the merging of the two bilayers in the contact zone (see Fig. S2.B in the Supplementary Information) and has the same overall morphology as the adhered state. These different pathways lead to the morphology diagram as shown in Fig. 3, which summarizes the outcomes of 93 independent simulation runs or fusion attempts. The planar membrane's inital molecular area is plotted along the $x$ axis and the vesicle's initial molecular area along the $y$ axis; these two quantities determine the initial tensions in these two membranes, respectively.

We define the fusion regime to contain all initial tension values for which we observed at least one successful fusion event. This regime contains 74 of our 93 independent fusion attempts. In Fig. 3, the fusion regime is bounded from above by the dashed rupture lines at which the planar membrane or the vesicle rupture prematurely before the system can explore any other pathway. Note that the vesicle ruptures at a smaller molecular area (or, equivalently, a smaller tension) than the planar membrane patch as a result of the weakening effect of the vesicle curvature on the membrane stability. As one starts from the rupture (dashed) lines and decreases the initial tensions, one moves across the fusion regime (shaded area) until one reaches the dotted line in Fig. 3, which corresponds to the stability line of the hemifused state.

Within the fusion regime, the time evolution of the membrane morphology is stochastic and can follow different pathways starting from the same values $(x, y)$ for the two initial tensions. Indeed, the fusion regime displayed in Fig. 3 contains 42 successful fusion events, eight vesicle ruptures, three planar membrane ruptures, and 21 hemifusion events. The blue squares within the shaded fusion regime correspond to those initial tension values for which fusion represents the dominant process. The detailed statistics of all simulation runs included in Fig. 3 is given in Table S1 of the Supplementary Information, which also contains additional illustrations of the different pathways within the fusion regime.

Inspection of Fig. 3 shows that the bilayer membranes simulated here are more stretchable than phospholipid bilayers and behave, in

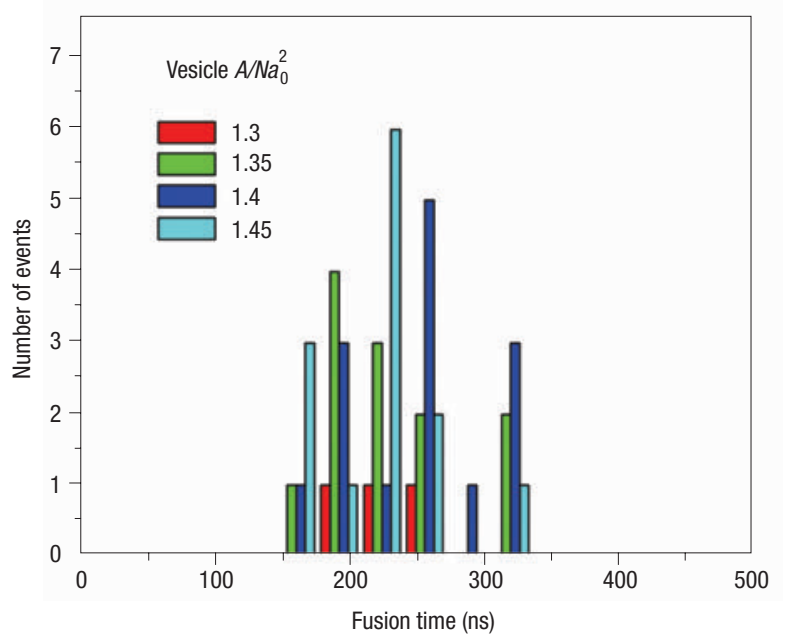

Figure 4 Histogram of the $\mathbf{4 2}$ out of $\mathbf{9 3}$ independent simulations that result in a fusion pore appearing in a given time. Results are grouped according to the vesicle's projected molecular area, $A / \mathrm{Na}_{0}{ }^{2}$ (the rows in Fig. 3). For the cases with $A / \mathrm{Na}_{0}{ }^{2}=1.3$, a total of 17 simulations were performed, three of which resulted in fusion within $1.6 \mu \mathrm{s}$, two cases ended in the planar membrane rupturing, and the remaining 12 cases ended in hemifusion. For $A / \mathrm{Na}_{0}{ }^{2}=1.35,25$ simulations were performed, of which 12 resulted in fusion, one in adhesion of the vesicle, one in premature rupture of the planar membrane, and the remaining 11 cases in hemifusion. For $A / \mathrm{Na}_{0}{ }^{2}=1.4,30$ simulations were performed, of which 14 resulted in fusion, two in adhesion, one in premature rupture of the vesicle and the remaining 13 cases resulted in hemifusion. For $A / N a_{0}{ }^{2}=1.45$, a total of 21 simulations were performed, of which 13 resulted in fusion, one in hemifusion and in the remaining seven cases the vesicle ruptured. Note that all simulations were run for at least $640 \mathrm{~ns}$, and many for 1,600 ns, ensuring that every case was simulated for at least twice as long as the largest observed fusion time.

this respect, more like block-copolymer bilayers ${ }^{21}$ (see also Fig. S1 of the Supplementary Information). Thus, in order to apply the morphology diagram in Fig. 3 to phospholipid bilayers, one should rescale both the $x$ and the $y$ axis in such a way that the maximal molecular areas correspond to the experimentally observed ones.

Each fusion event can be characterized by its fusion time, which is the time from first membrane contact to the appearance of a complete fusion pore. The measured fusion times for the 42 successful fusion events are shown in Fig. 4. This histogram has two remarkable features. First, the fusion-time distributions for different vesicle tensions have significant overlap and are all centred around 200-300 ns. Second, we have not observed any fusion time between $350 \mathrm{~ns}$ and $2 \mu \mathrm{s}$. This upper cut-off of the fusion-time distribution arises from the stabilization of the hemifused state in the membrane geometry considered here. Because the hemifused state is (meta)stable for relatively large initial tensions, fusion can only occur at even larger tensions for which the fusion pathway exhibits no activation barrier. Similar fusion-time distributions should apply to the fusion of two vesicles that are comparable in size. In general, the stabilization of the hemifused state will depend on the membrane areas that are stretched initially. If the area of the planar membrane is large compared with the vesicle area, relatively stable hemifused intermediates are also observed if one initially stretches only a subsegment of the planar membrane. On the other hand, if one stretches such a larger membrane uniformly, the hemifused states are only stabilized for smaller initial tensions, and the dotted stability line in Fig. 3 is shifted towards smaller tension values. 
This leads to an increased fusion regime, and one can then explore fusion pathways that exhibit an appreciable, tension-dependent activation barrier, and thus are characterized by longer fusion times.

Our observations imply that fusion induced by relatively large tensions occurs within $350 \mathrm{~ns}$ but represents a stochastic and unreliable process. In order to obtain a more reliable fusion protocol, it seems necessary to apply fine-tuned forces that are localized in space and last over a certain period of time. We have explored such protocols in order to mimic the localized forces that membrane anchored proteins may exert ${ }^{22}$ during the fusion of biomembranes (J. C. Shillcock and R. Lipowsky, in preparation). In fact, distinguishing between the different models for the action of fusion proteins $s^{4,5}$ is a compelling target for further DPD simulation studies. It is possible to measure the work performed during the fusion process in a DPD simulation, as has recently been done in coarse-grained molecular dynamics simulations ${ }^{9}$, and to relate this to experimental estimates of the forces exerted by fusion proteins ${ }^{23}$. It should also be possible to calibrate the DPD parameters by extending a method that has recently been applied to coarsegrained molecular dynamics simulations ${ }^{21}$. In general, our study shows that particle-based DPD simulations can reveal molecular rearrangements on relatively large length- and timescales that are relevant to many biomimetic and biological processes.

Received 18 June 2004; accepted 23 December 2004; published 13 February 2005

References

1. Alberts, B. et al. Molecular Biology of the Cell 2nd edn (Garland, New York, 1989).

2. Lasic, D. D. in Vesicles (ed. Rosoff, M.) Ch. 10 (Surfactant Science Series vol. 62, Marcel Dekker, New York, 1995).

3. Shillcock, J. C. \& Lipowsky, R. Equilibrium structure and lateral stress distribution of amphiphilic bilayers from dissipative particle dynamics. J. Chem. Phys. 117, 5048-5061 (2002).

4. Jahn, R. \& Grubmüller, H. Membrane fusion. Curr. Opin. Cell Biology 14, 488-495 (2002).

5. Mayer, A. Membrane fusion in eukaryotic cells. Annu. Rev. Cell. Dev. Biol. 18, 289-314 (2002).

6. Lindau, M. \& de Toledo, G. A. The fusion pore. Biochim. Biophys. Acta 1641, 167-173 (2003).

7. Ohta-lino, S. et al. Fast lipid disorientation at the onset of membrane fusion revealed by moleculer
Dynamics simulations. Biophys. J. 81, 217-224 (2001).

8. Marrink, S. J. \& Mark, A. E. The mechanism of vesicle fusion as revealed by molecular dynamics simulations. J. Am. Chem. Soc. 125, 11144-11145 (2003).

9. Stevens, M. J., Hoh, J. H. \& Woolf, T. B. Insights into the molecular mechanism of membrane fusion from simulation: evidence for the association of splayed tails. Phys. Rev. Lett. 91, 188102 (2003).

10. Chanturiya, A., Scaria, P., Kuksenok, O. \& Woodle, M. C. Probing the mechanism of fusion in a twodimensional computer simulation. Biophys. J. 82, 3072-3080 (2002).

11. Noguchi, H. \& Takasu, M. Fusion pathways of vesicles: a Brownian dynamics simulation. J. Chem Phys. 115, 9547-9551 (2001).

12. Siegel, D. P. The modified stalk mechanism of lamellar/inverted phase transitions and its implications for membrane fusion. Biophys. J. 76, 291-313 (1999).

13. Markin, V. S. \& Albanesi, J. P. Membrane fusion: stalk model revisited. Biophys. J. 82, 693-712 (2002).

14. Mueller, M., Katsov, K. \& Schick, M. A new mechanism of model membrane fusion determined from Monte Carlo simulation. Biophys. J. 85, 1611-1623 (2003).

15. Hoogerbrugge, P. J. \& Koelman, J. M. V. A. Simulating microscopic hydrodynamic phenomena with dissipative particle dynamics. Europhys. Lett. 19, 155-160 (1992).

16. Kranenburg, M., Venturoli, M. \& Smit, B. Molecular simulations of mesoscopic bilayer phases. Phys. Rev. E. 67, 060901 (2003).

17. Yamamoto, S., Maruyama, Y. \& Hyodo, S. Dissipative particle dynamics study of spontaneous vesicle formation of amphiphilic molecules. J. Chem. Phys. 116, 5842-5849 (2002).

18. Groot, R. D. \& Warren, P. B. Dissipative particle dynamics: bridging the gap between atomistic and mesoscopic simulation. J. Chem. Phys. 107, 4423-4435 (1997).

19. Vattulainen, I., Karttunen, M., Besold, G. \& Polson, J. M. Integration schemes for dissipative particle dynamics simulations: from softly interacting systems to hybrid models. J. Chem. Phys. 116, 3967-3979 (2002)

20. Warren, P. B. Dissipative particle dynamics. Curr. Opin. Colloid Interface Sci. 3, 620-624 (1998).

21. Nielsen, S. O., Lopez, C. F., Srinivas, G. \& Klein, M. L. Coarse grain models and the computer simulation of soft materials. J. Phys. Chem. B. 108, 8153-8160 (2004).

22. McNew, J. A. et al. Close is not enough: SNARE-dependent membrane fusion requires an active mechanism that transduces force to membrane anchors. J. Cell Biol. 150, 105-117 (2000).

23. Yersin, A. et al. Interactions between synaptic vesicle fusion proteins explored by atomic force microscopy. Proc. Natl Acad. Sci. USA 100, 8736-8741 (2003).

\section{Acknowledgements}

We thank Erwin Neher for helpful correspondence and acknowledge support by the Human Frontier Science Project through research grant RGP0072/2003. We also thank one of the reviewers for their rather detailed comments on the first version of this manuscript.

Correspondence and requests for materials should be addressed to R.L.

Competing financial interests

The authors declare that they have no competing financial interests. 